\title{
A theoretical treatment of void electromigration in the strip geometry
}

\author{
M. Ben Amar *, L.J. Cummings, G. Richardson \\ Laboratoire de Physique Statistique de Ecole Normale Supérieure associe au C.N.R.S. et Univ. Paris VI et VII, 24 rue Lhomond, \\ 75231 Paris Cedex 05, France
}

\begin{abstract}
The void electromigration process in the strip geometry is investigated analytically and numerically. The void is assumed to travel either along the axis of symmetry of the metal strip or at the boundary. In each case, the shape, the velocity of the void and the characteristic electrical current are predicted. (c) 2000 Published by Elsevier Science B.V. All rights reserved.
\end{abstract}

PACS: 66.30.Qa; 68.35.Ja; 85.40.Qx; 03.40.Gc

\section{Introduction}

Metal conductors subjected to intense electrical currents can exhibit the phenomenon of surface electromigration. A void can form within the conductor (nucleating either in the interior or at a boundary) and then "drift" within the conductor, changing its shape as it does so. Since the conductors are thin, the problem is almost twodimensional (2D). Typical experimental configurations include the case in which the lower conductor boundary is set on some rigid insulating substrate with the upper boundary open to the air (assumed insulating), and the case in which both boundaries are adjacent to rigid insulators. This drift of voids within conductors is due to the diffusion of atoms from one part of the void boundary to another, driven both by the electric

\footnotetext{
${ }^{*}$ Corresponding author.

E-mail address: benamar@lps.ens.fr (M. Ben Amar).
}

field and by interfacial tension (which causes the atoms to move along the boundary to positions of large curvature).

The problem is of interest because such migration of voids within conductors is a relatively common form of circuit failure in electrical components. Experimental evidence suggests that for a long thin conductor subjected to a uniform electric field at its ends, a void at the conductor boundary will undergo fairly steady translation for a time, with a steepening profile, until some critical void shape is attained. At this point the void motion becomes visibly unstable; a narrow finger emerges from the void and propagates transversely to the electric field until it has traversed the conductor height, and circuit failure occurs [1]. Damage of this kind can be suppressed by covering the exposed surfaces of the conductor with a layer of glass ("passivating") but in this case approximately circular voids nucleate in the interior of the conductor, and these can again deform and cause failure. Both geometries (voids at a conductor 
boundary, and voids in the interior of a conductor) are therefore of interest and investigated in this paper.

Most of the existing literature on this problem is either experimental [1,3,7,11,14], numerical simulations $[2,12,13,18]$, or concerned with circular or nearly circular voids translating in infinite conducting media [5].

The layout of this paper is as follows. We begin by setting out the mathematical model in Section 2 , and develop a complex variable formulation in terms of conformal mappings to numerically solve the steady propagation of voids, first inside the metal strip, and then at the boundary. In particular, we show that steady propagating soliton-like voids do not exist. To treat unsteady distortion of the boundary, we prefer an approach similar to that used to describe surface waves on inviscid fluid of finite depth. In the appropriate parameter regime we find that 'long-wavelength' voids are described by a nonlinear fourth-order differential equation, which must then be solved numerically. Several solution types are presented. Finally, we discuss the implications of our results and present our conclusions.

\section{The model}

Consider a single crystal metal film thin enough so that it may be considered to be effectively $2 \mathrm{D}$. The upper and lower edges of the film are the lines $y= \pm a$. A constant electrical current $I=2 a \Sigma E_{\infty}$ flows through the metal film from $x=+\infty$ to $x=-\infty$, where $E_{x} \rightarrow-E_{\infty}$ ( $E$ being the electric field) for $x \rightarrow \infty$ and $\Sigma$ is the conductivity of the film. After a typical nucleation time, not considered here, a void with boundary $\Gamma$ has formed and moves due to the imposed electric current. The electrical potential $\phi$ satisfies the 2D Laplace equation

$\nabla^{2} \phi=0 \quad$ with $\vec{E}=-\nabla \phi$

within the film. No current flows through the film edges, so that

$E_{n} \equiv \vec{E} \cdot \hat{n}=0 \quad$ on the void $\Gamma$

and $E_{y}=0$ for $y= \pm a$.
The contour $\Gamma$ moves because surface self-diffusion and electromigration produce a mass current along it. For simplicity, we assume that the surface tension $\gamma$ and adatom mobility $M$ are to a good approximation isotropic. Let $\theta=\hat{\theta}(S)$ (where $S$ is the arclength along $\gamma$ ) be the turning angle of an arbitrary point on $\Gamma$ (Fig. 1). (Note that $-\pi / 2 \leqslant \hat{\theta}(S) \leqslant 0$ for $S \geqslant 0$.) The point on the void edge moves with a normal velocity

$v_{n}=-\partial_{S} J=-\left(-M \Omega \gamma \partial_{S}^{2} \kappa+Z^{*} e M \partial_{S} E_{S}\right)$,

where $E_{S}$ is the tangential component of the electric field, $\Omega$ the atomic volume, and $Z^{*} e$ is the effective charge of a metal ion [5]. The sign of the curvature $\kappa$ is chosen to be positive if the center of curvature lies within the void. With this convention, $\kappa$ is positive in the figure and $\kappa=\partial_{S} \hat{\theta}$. Physically, this curvature term indicates that the atoms diffuse toward positions with large curvature. The sign of $Z^{*} e$ is not necessarily positive: when the surface diffusion of the ions is dominated by the collisions with the electrons (the so-called electron wind) the effective charge becomes negative. In this case, the analysis still applies, but one has to reverse the sign of the imposed electric field; the void migration follows the imposed current if the effective charge of the ions is negative, and the void moves in the opposite direction otherwise. In this part, we assume that the film edges at $y= \pm a$ are fixed to an insulating material; the edges therefore do not deform as time passes. In the second part of the paper, the distortion of one border will be considered.

We will seek the steady state "bubble shape" void which travels along the axis of symmetry of the strip with constant velocity $U$. We employ a

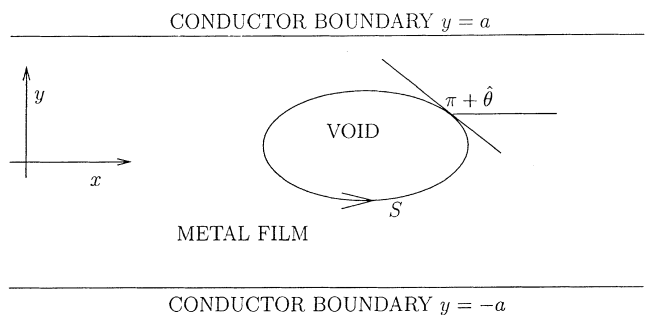

Fig. 1. Diagram of void electromigration within a conductor metal strip. 
moving frame of reference in which the void's center is at the origin and set $\phi(0)=0$. For convenience, we assume that $Z^{*} e>0$ and introduce the dimensionless variables $\tilde{x}=x / a, \tilde{y}=y / a, \tilde{E}=$ $E / E_{\infty}$, and $\tilde{t}=Z^{*} e M E_{\infty} t / a^{2}$. From this point on, we drop the tildes. In dimensionless units, the boundary condition to be applied along the void is $\frac{\partial E_{S}}{\partial S}-\alpha \frac{\partial^{2} \kappa}{\partial S^{2}}=U \sin \theta_{T}=-U \frac{\partial y}{\partial S} \quad$ on the void,

with

$\alpha=\frac{\Omega \gamma}{Z^{*} e E_{\infty} a^{2}}$

and $U$ the dimensionless parameter such that

$v=U \frac{Z^{*} e M E_{\infty}}{a}$.

From a theoretical point of view electromigration belongs to the same class of Laplacian free boundary problem as the viscous fingering instability [8-10]. The numerical and theoretical methods are standard by now. For the steady growth, we have adapted the conformal mapping technique of McLean and Saffman [4] explained in the next section.

\subsection{Conformal mapping}

In a previous paper, one of us has stressed the similarities between electromigration and the Saffman-Taylor (ST) instability in the strip, in the steady case. In particular, the case of an infinite void was especially surprising since a selected width of $2 / 3$ compared to the strip width was found numerically without surface tension. From an experimental point of view, it is perhaps more realistic to consider finite-sized voids. In viscous flow, they have been less studied both experimentally [6] but also analytically [15-17]. To numerically find the shape and the velocity of the void numerically, we choose the conformal mapping technique of McLean and Saffman [4] which is in fact a hodograph method. Instead of working in the laboratory space, we use the plane of the complex potential $\Phi=\phi+\mathrm{i} \chi$ and define the $\sigma$-plane by $\sigma=s+\mathrm{i} t=\exp \pi\left(\left[\phi-\phi_{0}+\mathrm{i} \chi\right]\right)$.

Recall that

$E_{x}=E_{\infty}=-1, \quad E_{y}=0 \quad$ as $x \rightarrow \mp \infty$

and $-1<y<1$.

So

$\phi \equiv x \quad$ as $x \rightarrow \mp \infty, \quad$ and $\quad-1<y<1$.

In the $\sigma$-plane the bubble occupies a segment: $\sigma=s$ with $s \in\left[1, s_{0}^{2}\right]$. For symmetric bubbles (right-left symmetry or symmetry with respect to the $y$-axis), the potential $\phi$ is an odd function of $x$ so the middle of the bubble is represented by $s=s_{0}=\exp \left(-\pi \phi_{0}\right)$. At this point, the tangent to the bubble is horizontal. But with capillarity, this symmetry is destroyed and the point of horizontal tangent is no longer at the origin, middle of the bubble. In this case, we will parametrize it by $s=s_{1}$. The growth $x$-axis is transformed into $[1, \infty]$, and the upper part of the strip: $(y=1)$ into the negative $s$ axis: $[-\infty, 0]$. The complex electric field becomes

$q \exp [-\mathrm{i}(\theta+\pi)] \equiv-\frac{1}{E} \frac{\mathrm{d} \Phi}{\mathrm{d} z}$,

where $E$ is the magnitude of the electric field at the top of the void: $y=\mp \lambda,\left(s=s_{1}\right)$ corresponding to the middle when it has the right-left symmetry. The growth rate of the void $U$ becomes $U=E / \lambda, q$ is the solution of

$\beta q s \frac{\mathrm{d}}{\mathrm{d} s}\left(q s \frac{\mathrm{d} \theta}{\mathrm{d} s}\right)+q=\frac{y}{\lambda}$,

with $\beta=\pi^{2} \alpha E$. The analyticity of $z$ as a function of $\Phi$ or $\sigma$ gives the useful relation for the shape

$z=x+\mathrm{i} y=-\frac{1}{E \pi} \int_{s}^{1} \frac{\exp (\mathrm{i} \theta)}{q\left(s^{\prime}\right) s^{\prime}} \mathrm{d} s^{\prime}+\frac{L}{2}$.

So the length $L$ of the void and its width $\lambda$ are given by

$L=\frac{2}{E \pi} \int_{s_{0}^{2}}^{1} \frac{\cos \theta}{q\left(s^{\prime}\right) s^{\prime}} \mathrm{d} s^{\prime}$, 
with

$\lambda=-\frac{1}{E \pi} \int_{s_{1}}^{1} \frac{\sin \theta}{q\left(s^{\prime}\right) s^{\prime}} \mathrm{d} s^{\prime}=-\frac{1}{E \pi} \int_{s_{1}}^{s_{0}^{2}} \frac{\sin \theta}{q\left(s^{\prime}\right) s^{\prime}} \mathrm{d} s^{\prime}$.

Finally, the Cauchy relation between the real and imaginary part of $\ln (q \exp (-\mathrm{i} \theta))$ on the $s$-axis gives:

$\ln q(s)=-\frac{\left(s-s_{1}\right)}{\pi}$ P.P. $\int_{s_{0}^{2}}^{1} \frac{\theta\left(s^{\prime}\right)}{\left(s^{\prime}-s_{1}\right)\left(s^{\prime}-s\right)} \mathrm{d} s^{\prime}$,

where P.P. means that the principal part of the integral is considered.

\subsection{Finite voids without curvature effects}

Combining the interface condition: $q=y / \lambda$ with Eqs. (12) and (15), we can define a unique integral equation for the finite-size void. Noting that $/ S s_{1}=s_{0}$ for this case, one finds

$$
\begin{aligned}
\frac{1}{2} \ln & {\left[1+\frac{2}{E \lambda \pi} \int_{s}^{1} \frac{\sin \theta\left(s^{\prime}\right)}{s^{\prime}} \mathrm{d} s^{\prime}\right] } \\
& =-\frac{\left(s-s_{0}\right)}{\pi} \text { P.P. } \int_{s_{0}^{2}}^{1} \frac{\theta\left(s^{\prime}\right)}{\left(s^{\prime}-s_{0}\right)\left(s^{\prime}-s\right)} \mathrm{d} s^{\prime} .
\end{aligned}
$$

This integral equation appears to have two independent parameters: the product $E \lambda$ (equivalent to $U \lambda$ for the ST problem [8-10]) and $s_{0}$, but in fact these two parameters are connected, contrary to the ST instability. Only one parameter can be varied freely for example $s_{0}$, then the bubble size is completely fixed and also the electric field.

For $s_{0}$ values slightly less than 1, infinitely small voids are expected. For viscous flow, this corresponds to ellipsoidal bubbles [15-17] with a relative velocity (compared to the velocity at infinity) varying from 1 to 2 , the latter limit corresponding to circular bubbles. These circular bubbles are in fact the solution of the infinite geometry, being so small that they do not feel the strip-geometry. In this limit of circular patterns of small radius $R$ for the electromigration problem, the growth of the void can be solved explicitly

$$
\begin{aligned}
z= & -\frac{1}{E(1-E)}\left(E^{2} R^{2}+(\phi+\mathrm{i} \chi-E z)^{2}\right)^{1 / 2} \\
& -\frac{\phi+\mathrm{i} \chi-E z}{E} .
\end{aligned}
$$

On the circular void with $\lambda=R, R$ being the radius, one has: $\phi=E x$ and $\chi=0$ which is compatible with the boundary condition $y=\lambda q$. So on the void one obtains

$z=-\frac{R}{(1-E)}\left(1-\frac{y^{2}}{R^{2}}\right)^{1 / 2}+\mathrm{i} y$,

which proves that $E$ is selected and is equal to 2 . As a consequence, the velocity is $2 / R$, a rather classical result [5].

Another limit concerns that of large voids given by $s_{0} \rightarrow 0$ : in this case, characteristic values such as $\lambda$ and $E \lambda$ (with $\lambda=0.66$, the limiting $\lambda$ value of an infinite void [2] and $E \lambda=2$ giving $E=3$ ) must be obtained numerically. It turns out that we have found that $E$ varies from 2 up to 3 (see Fig. 2); $\lambda$, the half width of the void varies from 0 up to 0.66 , and the product $E \lambda$ varies from 0 to 2 . Somewhat surprisingly, we note that the void remains almost circular: even when the width occupies half the strip, the length $L$ remains close to $2 \lambda$ as confirmed by Fig. 3. Note that the ratio $L / 2 \lambda$ is always greater than 1. Finally, the velocity of the void is rather easy to predict; its value is given by: $U=E / \lambda$. Since $E$ varies from 2 to 3 and $\lambda$ from 0

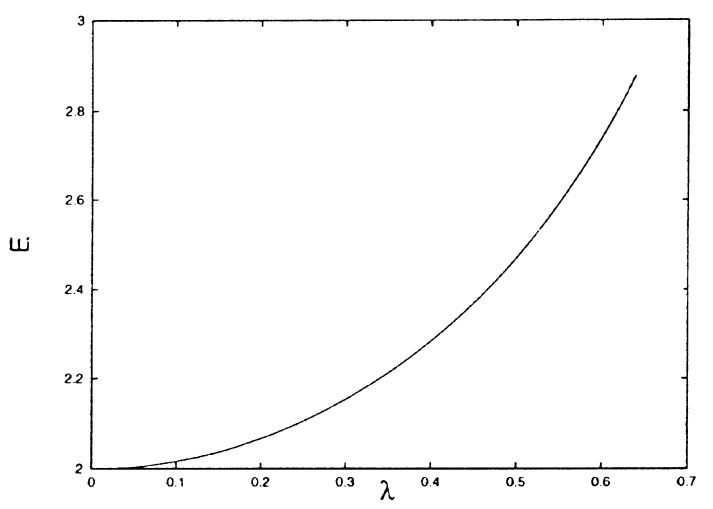

Fig. 2. The electric field calculated at the top of the void versus its half width $\lambda$. 


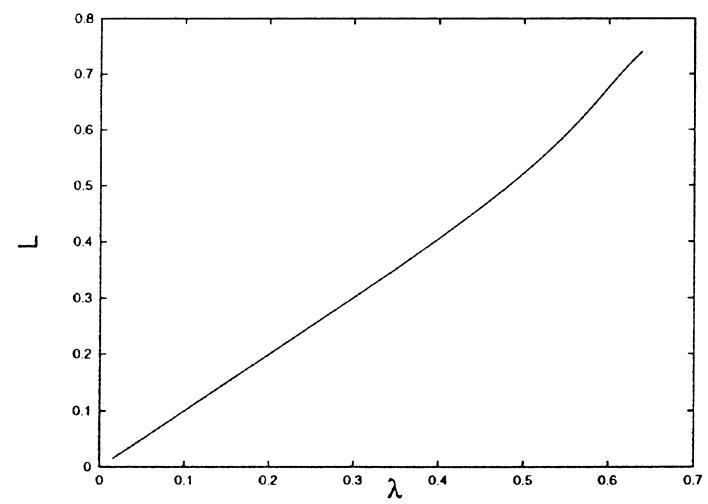

Fig. 3. Half the length of the void versus half the width $\lambda$.

to $2 / 3$, the growth rate is a rapidly decreasing function of the void size as shown in Figs. 4 and 5.

\subsection{Finite voids with capillarity}

Previously we have shown that, in the absence of surface tension, voids of arbitrary length can exist. Once the length is fixed, all other characteristics of the growth are determined such as the complete shape of the void (and hence its width), the ratio between the electric field at the widest point of the void and the electric field at infinity, and of course the growth rate $U$. In viscous fingering surface tension is responsible for the selection process, that is, it selects $\lambda$ once the length of the bubble is fixed. The bubbles appear elongated from the limit of an infinite finger to the limit of a

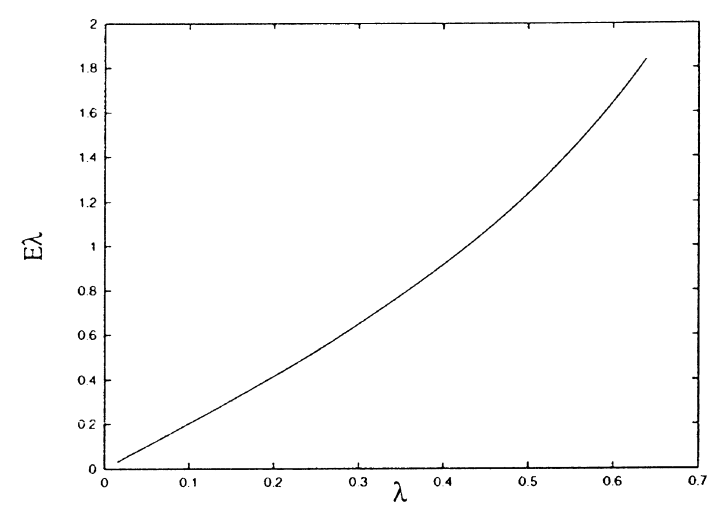

Fig. 4. The eigenvalue " $E \lambda$ " versus the half width $\lambda$.

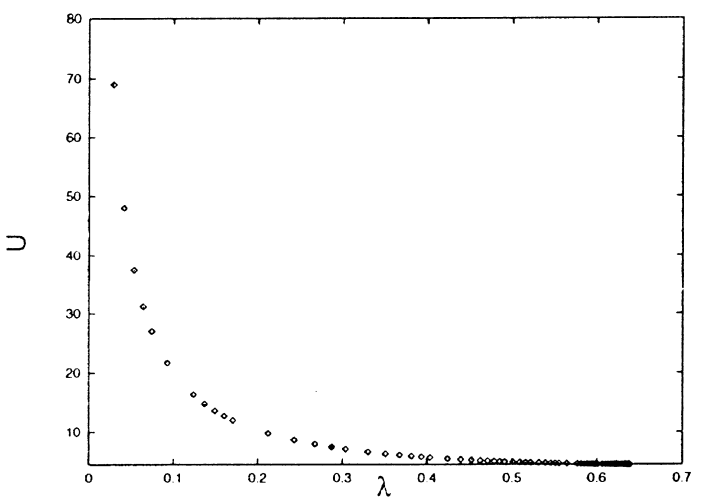

Fig. 5. The growth rate versus the relative width $\lambda$ of the void.

tiny circular bubble. This means that here also the ratio $L / 2 \lambda$ is always greater than 1 .

For the electromigration problem, selection comes from the conditions of existence, and one might wonder what will be the role of the equivalent capillarity. It turns out that the effect of surface tension is entirely different. Note that 'surface tension' does not act on a completely circular void, whatever its strength. At fixed surface tension, increasing the length of the void from 0 (which is still possible in some limit), one notices that the half width increases remaining very close to half the length. So the void remains circular in shape and the electric field $E$ still has a value close to 2 . At some typical length for the void, this solution seems to disappear, although we have no rigorous proof of it. The only thing we can say is that we cannot find solutions above some critical length $L_{0}(\alpha)$ which depends on the value of the capillary coefficient $\alpha$. Two other surprising results must also be stressed. First, when one increases the surface tension from $10^{-2}$ up to 10 , for example, the numerical results are still very close indicating the poor influence of surface tension as shown by Fig. 6. Secondly, although slightly elongated, the voids are only weakly elongated along the $y$-axis, contrary to what is found for viscous drops and for voids without capillarity. This indicates that since $\lambda$ is geometrically restricted to 1 , and since $L$ will remain smaller than $2 \lambda$, there is no chance of observing an infinite void in this geometry. This explains why the infinite finger cannot exist in presence of surface tension [2]. Surface tension 


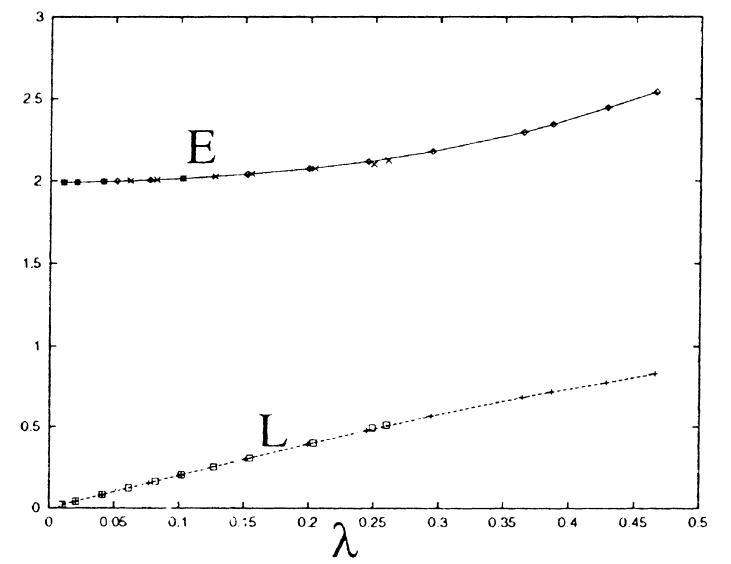

Fig. 6. The electric field $E$ and the length $L$ of the void versus $\lambda$ for two values of the capillary coefficient $\alpha: \alpha=10$ (continuous line), and $\alpha=0.01$ (crosses and squares). Note that solutions cannot be obtained numerically above a typical threshold for the $\lambda$ value, which itself depends on $\alpha$.

prevents the growth of large structures and thus really plays a stabilizing role.

\section{Voids at a conductor boundary}

We consider now the distortion of the lower edge of the film $(y=0$, when the current is switched off) and we look for steady propagation of a soliton-like void, the other edge (line $y=a$ ) remaining stuck. Although we are seeking a 'soliton' void of finite extent, the easiest treatment involves the whole edge, with perhaps exponentially decreasing tails. In the last part of this paper, a weakly nonlinear analysis derived from standard asymptotic methods similar to those used in hydrodynamics is proposed. The previous conformal mapping method can be adapted without difficulty. The void boundary $\Gamma$ will be oriented like the $x$ axis, and the arclength along it $(S)$ will be taken to be 0 at the top of the void. As previously, the void is a 'streamline' and is assumed to be the whole lower edge. $\chi$ has a constant value on the boundaries where the normal component of the electric field vanishes. On the distorted border, we choose $\chi=0$. Since at $+\infty, \chi$ is equal to $y$, one deduces that $\chi=1$ when $y=1$. With the $\sigma$-mapping defined by $\sigma=\exp \pi[\phi+\mathrm{i} \chi]$, the void is transformed into the positive half $s$-axis: $\sigma=s$, where $s \in[0, \infty]$, and the upper part of the strip: $y=1$ into the negative $s$-axis: $[-\infty, 0]$. Here, we choose as the modulus of the electrical field $q^{\prime}=E q$ where $E$ is the value at the top of the void. Taking into account the conditions at infinity, Eq. (11) is transformed into

$\alpha \pi^{2} q^{\prime} s \frac{\mathrm{d}}{\mathrm{d} s}\left(q^{\prime} s \frac{\mathrm{d} \theta}{\mathrm{d} s}\right)+q^{\prime}=V \frac{y}{\lambda}+1$,

with $\lambda$ being the relative height of the void compared to the width of the strip and $V=(U \lambda)$. From Eq. (19), one observes that in the absence of the surface tension, the maximum value of the electric field $q^{\prime}$ is obtained at the location on the border of the maximum height of the void. One now needs to relate $y$ to $s$ as previously for Eq. (12)

$z=x+\mathrm{i} y=\mathrm{i} \lambda+\frac{1}{\pi} \int_{1}^{s} \frac{\exp (\mathrm{i} \theta)}{q^{\prime}\left(s^{\prime}\right) s^{\prime}} \mathrm{d} s^{\prime}$,

which implies that

$\int_{1}^{0} \frac{\sin (\theta)}{q^{\prime}\left(s^{\prime}\right) s^{\prime}} \mathrm{d} s^{\prime}=\int_{1}^{\infty} \frac{\sin (\theta)}{q^{\prime}\left(s^{\prime}\right) s^{\prime}} \mathrm{d} s^{\prime}=-\lambda$.

Now, we must relate $q^{\prime}$ to $\theta$, by using the Cauchy relation between the real and imaginary part of $\ln \left[q^{\prime} \exp (-\mathrm{i} \theta)\right]$. Note that, on the real $s$-axis, $\theta$ vanishes for $s<0$, and so we obtain

$\ln q^{\prime}(s)=-\frac{1}{\pi}$ P.P. $\int_{0}^{\infty} \frac{\theta\left(s^{\prime}\right)}{\left(s^{\prime}-s\right)} \mathrm{d} s^{\prime}$.

Moreover we require that $\int_{0}^{\infty}(\theta(s) / s) \mathrm{d} s=0$, which is obviously true for a symmetric bump.

One can now prove by contradiction that this set of equations has no solution in the absence of surface tension. To obtain the contradiction, we first define an upper bound for $\lambda$ using the theorem of the extremum of the modulus of an analytical function. We know that the extrema of the modulus are located on a boundary. If we consider $q^{\prime}=|\mathrm{d} \Phi / \mathrm{d} z|$, its maximal value is attained on the border for $y=\lambda$, at the top of the bump. Consider now $\int E_{x} \mathrm{~d} y$ along a vertical line going from one border to the other. This quantity is independent of $x$ and its value at $\pm \infty$ is simply 1 . So 


$$
\begin{aligned}
\int_{0}^{1} E_{\infty, x} \mathrm{~d} y=1= & \int_{y=\lambda}^{1} E_{x} \mathrm{~d} y \leqslant \int_{y=\lambda}^{1}\left|\frac{\mathrm{d} \Phi}{\mathrm{d} z}\right| \mathrm{d} y \\
& \leqslant(1-\lambda)(1+V),
\end{aligned}
$$

which implies

$\lambda \leqslant \frac{V}{1+V}$.

We now establish a lower bound for $\lambda$ using the asymptotics at $x= \pm \infty$ of the previous equations. Since $\theta$ vanishes at both ends, one can assume $\theta \sim \theta_{0} s^{\tau}$ as $s \rightarrow 0, \theta \sim-\theta_{0} s^{-\tau}$ as $s \rightarrow \infty$ and $\tau$ being a positive number less than 1 . Taking into account Eqs. (20) and (19) one derives the behaviour of $q$ for $s \rightarrow 0$

$q=1+\frac{V}{\pi \lambda} \theta_{0} \frac{s^{\tau}}{\tau}$

and for $s \rightarrow \infty$ one finds

$q=1+\frac{V}{\pi \lambda} \theta_{0} \frac{s^{-\tau}}{\tau}$.

One can also derive the asymptotics of the righthand side of Eq. (22). For $s \rightarrow 0$, the leading behaviour is $\cot (\pi \tau) \theta_{0} s^{\tau}$ and for $s \rightarrow \infty$ it is $\cot (\pi \tau) \theta_{0} s^{-\tau}$ with the restriction $0<\tau<0.5$. So matching the asymptotic expansions, one obtains:

$\frac{V}{\lambda}=\pi \tau \cot (\pi \tau)$.

Since $\pi x \cot (\pi x)$ is always less than 1 for $0<x<0.5$, taking into account Eq. (24), one finally finds the following bounds for $\lambda$ :

$V \leqslant \lambda \leqslant \frac{V}{1+V}$,

which is of course impossible except in the limit $V \rightarrow 0$, as $\lambda \rightarrow 0$. This limit represents a linearization of the set of equations for small $\theta$ values, or the situation of a semi-infinite strip. Such solutions are immediately destroyed by the nonlinearities.

One might wonder if steady solutions can be restored with surface tension. There is little chance of recovering solutions at tiny surface tension, thus we examine the case of infinite surface tension directly using Eq. (4). If such solution exists, it would imply that there exists a threshold for $\alpha$ say $\alpha_{c}$ such that below $\alpha_{c}$, no solution appears, but above $\alpha_{c}$, with enough surface tension, a steady void can propagate. The limit $\alpha \rightarrow \infty$ implies that $U$ also goes to $\infty$ so that, to leading order, Eq. (4) reduces to:

$\frac{\partial^{3} \theta}{\partial S^{3}}=\frac{U}{\alpha} \sin \theta$.

The quantity $m=U / \alpha$ must be of order 1 for a nontrivial solution. It turns out that this equation has no solution which decays at $\pm \infty$. Multiplying both sides of Eq. (29) by $\partial \theta / \partial S$ and integrating one gets

$\left[\frac{\partial^{2} \theta}{\partial S^{2}} \frac{\partial \theta}{\partial S}\right]_{-\infty}^{\infty}-\int_{-\infty}^{\infty}\left(\frac{\partial^{2} \theta}{\partial S^{2}}\right)^{2} \mathrm{~d} S=m[-\cos (\theta)]_{-\infty}^{\infty}=0$.

Since $\theta$ vanishes at both extremities, this equation has no solution which damps at infinity, whatever the $m$-value. So clearly, even at strong surface tension, a steady void of "finite extent" with a bump-shape cannot propagate if one restricts to the physical model of electromigration presented above. The asymptotic analysis explained below arrives to the same conclusion: there is no solitonlike void which travels at constant velocity along the border. However, note that these voids, if they exist are not fatal.

\section{An asymptotic model for voids at a conductor boundary}

In this section we use asymptotic methods to derive a simplified model for the motion of a void at a conductor boundary. We return to the dimensional problem, writing $A=Z^{*} e M$ and $B=M \Omega \gamma$ for convenience. The problem is shown in Fig. 7, with a void on the upper boundary which is described by $y=\eta(x, t)$, with $\eta(\infty, t)=0$ and $\eta(-\infty, t)=2$.

Suppose the initial profile is of the form $\eta(x, 0)=\eta_{0} N(x / l)$, so that $l$ and $\eta_{0}$ are lengthscales of the disturbance in the $x$ - and $y$-directions, respectively. We then scale 


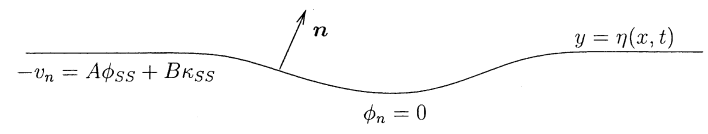

METAL FILM

$$
\nabla^{2} \phi=0
$$

CONDUCTOR BOUNDARY

$\phi_{n}=0$

Fig. 7. The full problem in the dimensional variables.

$x=l \bar{x}, \quad y=h_{0} \bar{y}, \quad \eta=\eta_{0} \bar{\eta}, \quad t=\frac{l \eta_{0}}{A E_{\infty}} \bar{t}$,

$\phi=E_{\infty} l \bar{\phi}$.

The appropriate time scale comes from the kinematic boundary condition, and depends on whether the electric field or surface tension dominates the void motion. Our choice assumes that the electric field effect either dominates, or is comparable with, the surface tension effect. We also introduce the two dimensionless parameters

$\epsilon=\frac{\eta_{0}}{h_{0}} \sim \frac{\text { "amplitude" }}{\text { "depth" }}, \quad \delta=\frac{h_{0}}{l} \sim \frac{\text { "depth" }}{\text { "wavelength" }}$.

This rescaling yields the dimensionless model (dropping overbars for convenience):

$$
\begin{aligned}
& \delta^{2} \phi_{x x}+\phi_{y y}=0 \quad-1<y<\epsilon \eta, \\
& \phi_{y}=\epsilon \delta^{2} \eta_{x} \phi_{x} \quad \text { on } y=\epsilon \eta, \\
& -\eta_{t}=\left(\partial_{x}+\epsilon \eta_{x} \partial_{y}\right)\left(\frac{\phi_{x}+\epsilon \eta_{x} \phi_{y}}{\left(1+\epsilon^{2} \delta^{2} \eta_{x}^{2}\right)^{1 / 2}}\right) \\
& +\gamma^{*} \frac{\partial}{\partial x}\left\{\frac{1}{\left(1+\epsilon^{2} \delta^{2} \eta_{x}^{2}\right)^{1 / 2}}\right. \\
& \left.\quad \times \frac{\partial}{\partial x}\left(\frac{\eta_{x x}}{\left(1+\epsilon^{2} \delta^{2} \eta_{x}^{2}\right)^{3 / 2}}\right)\right\} \quad \text { on } y=\epsilon \eta, \\
& \phi_{y}=0 \quad \text { on } y=-1, \\
& \phi_{x} \sim 1, \quad \eta \sim 0 \quad \text { as } x \rightarrow \infty,
\end{aligned}
$$

$\phi_{x} \sim \frac{1}{1+\epsilon \mathscr{Q}^{*}}, \quad \eta \sim \mathscr{Q}^{*} \quad$ as $x \rightarrow-\infty$,

where dimensionless coefficients $\mathscr{2}^{*}$ and $\gamma^{*}$ are defined by

$$
\mathscr{Q}^{*}=\frac{\mathscr{2}}{\eta_{0}}, \quad \gamma^{*}=\frac{B \eta_{0}}{A l^{3} E_{\infty}} \quad\left(\gamma^{*} \leqslant \mathrm{O}(1)\right) .
$$

We now assume that $\delta \ll 1$ and $\epsilon=1$, that is, a long-wavelength but deep void, and seek an asymptotic solution of the form $\phi=\phi^{(0)}+\delta^{2} \phi^{(1)}+$ $\mathrm{O}\left(\delta^{4}\right), \eta=\eta^{(0)}+\delta^{2} \eta^{(1)}+\mathrm{O}\left(\delta^{4}\right)$. Substitution in (31)-(35) at leading order yields:

$\phi^{(0)}=\phi^{(0)}(x, t)$,

$\eta_{t}^{(0)}=\phi_{x x}^{(0)}-\gamma^{*} \eta_{x x x x}^{(0)}$,

$\phi_{x}^{(0)} \sim 1, \quad \eta^{(0)} \sim 0 \quad$ as $x \rightarrow \infty$,

$\phi_{x}^{(0)} \sim \frac{1}{1+\mathscr{2}^{*}}, \quad \eta^{(0)} \sim \mathscr{2}^{*} \quad$ as $x \rightarrow-\infty$,

whilst at first order we find the following problem for $\phi^{(1)}$ :

$\phi_{y y}^{(1)}+\phi_{x x}^{(0)}=0$,

$\phi_{y}^{(1)}=0 \quad$ on $y=-1$,

$\phi_{y}^{(1)}=\eta_{x}^{(0)} \phi_{x}^{(0)} \quad$ on $y=-1$.

Integrating Eq. (41) between $y=-1$ and $y=\eta^{(0)}$ and applying Eqs. (42) and (43) gives

$\frac{\partial}{\partial x}\left(\phi_{x}^{(0)}\left(1+\eta^{(0)}\right)\right)=0$,

expressing conservation of electric current. We integrate (44), apply conditions (39) and (40) and use the result to eliminate $\phi_{x}^{(0)}$ from (38). Writing $f=\eta^{(0)}+1$ and $\lambda=1+2^{*}$ this yields the following parabolic nonlinear PDE for the free surface evolution:

$f_{t}+\left(\frac{1}{f}\right)_{x}+\gamma^{*} f_{x x x x}=0$,

$f(\infty, t)=1, f(-\infty, t)=\lambda$.

Thus we have a model with just one dimensionless parameter $\gamma^{*}$ which measures the relative effects of 
surface diffusion due to the surface tension and surface diffusion due to the electric field. The model is easily shown to conserve void area (when this is finite).

\subsection{The case of negligible surface tension}

Here the void motion may be approximated by Eq. (45) with $\gamma^{*}=0$, which is a first order nonlinear wave equation for $f$ with implicit solution

$f(x, t)=f_{0}\left(x+\frac{t}{f(x, t)^{2}}\right)$,

where $f_{0}$ is the initial void profile. Solutions are wave-like, with effective wavespeed $1 / f^{2}$; thus the void profile steepens since the deeper parts of the profile move faster. Hence these solutions exhibit finite-time blow-up, occurring when $f_{x}$ becomes infinite for some point on the profile.

Despite the general formula (46), it is difficult to find closed-form solutions for a realistic initial profile $f_{0}$. However, there is one initial (compact support) profile for which we can solve explicitly: this is the initially 'pointy' profile given by

$f_{0}(x)= \begin{cases}1, & |x|>1, \\ \sqrt{\lambda^{2}-x\left(1-\lambda^{2}\right)}, & -1<x<0, \\ \sqrt{\lambda^{2}+x\left(1-\lambda^{2}\right)}, & 0<x<1 .\end{cases}$

For $t>0$ the profile is still in three parts: $-1-t<x<s(t), s(t)<x<1-t$, where $s(t)$ is the position of the pointy tip; and the remainder of the $x$-axis, for which $f \equiv 1$. To find the void profile for $t>0$ we have to solve the following equation for $f$ :

$f=f_{0}\left(x+t / f^{2}\right)=\sqrt{\lambda^{2} \pm\left(1-\lambda^{2}\right)\left(x+t / f^{2}\right)}$

(which reduces to a quadratic for $f^{2}$ ) on the appropriate regions. The position of the tip $s(t)$ is then determined by the condition that the two parts of the profile meet up at this point. We find (choosing the sign of the square-root consistent with the initial data):

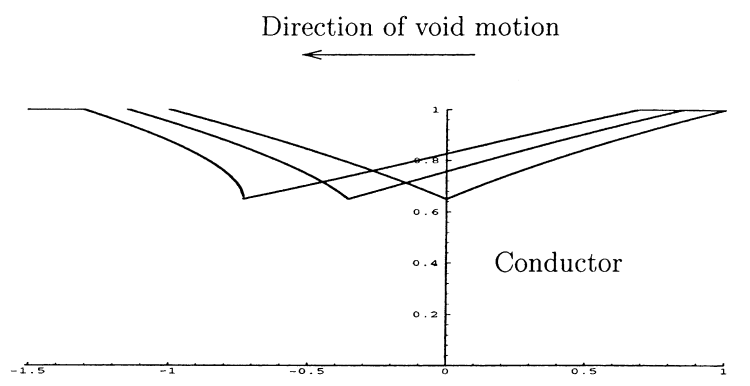

Fig. 8. Evolution of the ZST void solution (47) for $\lambda=0.65$. The profile is shown at times $t=0, t=0.15$ and $t=0.309 \approx t^{*}$.

$$
\begin{gathered}
\begin{aligned}
& f(x, t)= \frac{1}{\sqrt{2}}\left(\lambda^{2} \pm x\left(1-\lambda^{2}\right)\right. \\
&+\left[\left(\lambda^{2} \pm x\left(1-\lambda^{2}\right)\right)^{2}\right. \\
&\left.\left. \pm 4 t\left(1-\lambda^{2}\right)\right]^{1 / 2}\right)^{1 / 2}, \\
& s(t)<x< 1-t,-1-t<x<s(t), \\
& s(t)=-\frac{t}{\lambda^{2}} .
\end{aligned}
\end{gathered}
$$

Thus the deepest point of the void travels with constant speed, which means (since void speed $=1 / \mathrm{f}^{2}$ ) that the void maintains a constant depth. Blow-up occurs when $f_{x}$ first becomes infinite, which for this case happens at the point $x=s(t)^{-}$, at time $t^{*}=\lambda^{4} /\left(1-\lambda^{2}\right)$. The evolution of such a void for $\lambda=0.65$ is shown in Fig. 8.

\subsection{Numerical time-dependent solutions}

We have been unable to find any explicit solutions of eq. (45) for $\gamma^{*} \neq 0$, so we must solve it numerically. We present solutions with $f(+\infty)=$ 1 and $f(-\infty)=\lambda$ for the cases $\lambda<1, \lambda>1, \lambda=1$. We use a semi-implicit scheme (the $(1 / f)_{x}$ term is treated explicitly) based on the Crank-Nicolson scheme for the usual diffusion equation (the only complicating factor is that the system of matrix equations obtained is not tri-diagonal but 5-diagonal). Figs. 9 and 10 show the evolution of semiinfinite voids with initial profile

$f(x, 0)=\lambda+(1-\lambda)(\tanh x+1) / 2$, 


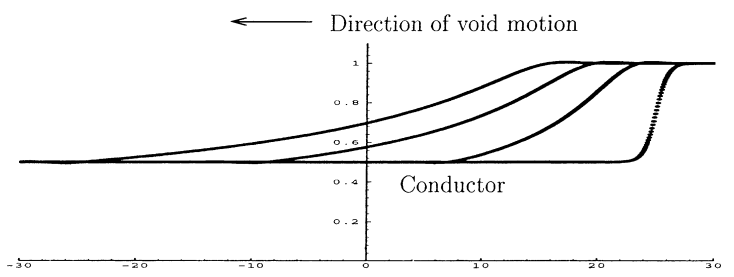

Fig. 9. Numerical solution of (45) for an initial profile $f(x, 0)=\lambda+(1-\lambda)(\tanh x+1) / 2(\lambda=0.5)$ and $\gamma^{*}=0.1$. The profile is shown at times $t=0,4,8,12$ (in the dimensionless units). Note the nonuniform scaling of horizontal and vertical axes, to make the void shape evolution easier to see.

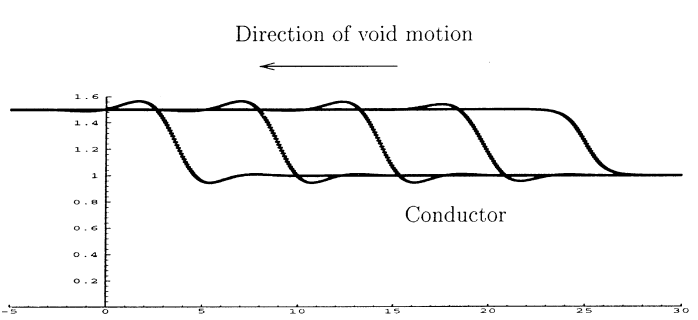

Fig. 10. Numerical solution of (45) for an initial profile $f(x, 0)=\lambda+(1-\lambda)(\tanh x+1) / 2(\lambda=1.5)$ and $\gamma^{*}=0.1$. The profile is shown at times $t=0,8,16,24,32$ (in the dimensionless units).

so that $f(+\infty, 0)=1$ and $f(-\infty, 0)=\lambda$. For Fig. $9 \lambda$ is 0.5 ; numerically this appears to be rather a stable situation, the steep initial profile smoothing out as time increases. Surface tension becomes less and less important for large times.

Fig. 10 shows the analogous solution for the case $\lambda=1.5$. The initial profile develops a small peak in advance of the moving disturbance and a small pit behind it, and the advancing wavefront steepens. It appears that this solution is approaching a travelling wave form; this is discussed further in Section 4.3. Finally, Fig. 11 shows a solution for which $f( \pm \infty)=1$. The initial disturbance is symmetric: $f(x)=1-0.6 \exp \left((x-25)^{2}\right)$.

If we take higher values of the surface tension parameter $\gamma^{*}$, the damping (stabilising) effect in Fig. 11 is severe (even with the value chosen it is significant); one has to take $\gamma^{*}$ quite small to get any kind of interesting behaviour. Nonetheless, the behaviour displayed in Figs. 10 and 11 is intrinsically different from that of the ZST $\left(\gamma^{*}=0\right)$ model of Section 4.1; in particular, with $\gamma^{*}>0$ the profile

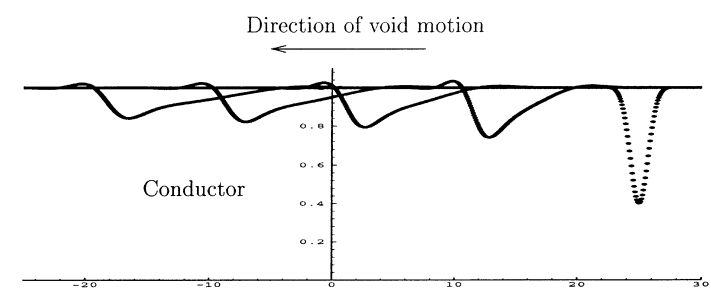

Fig. 11. Numerical solution of (45) for an initial profile $f(x)=1-0.6 \exp \left((x-25)^{2}\right)$ and $\gamma^{*}=0.1$. The profile is shown at times $t=0,8,16,24,32$ (in the dimensionless units).

cannot develop an actual shock. Fig. 9 does display the same characteristics as the ZST model though, and in fact the long-time asymptotics of such solutions are given by the ZST model.

\subsection{A travelling wave solution of (45) in the case} $\lambda>1$

We again assume boundary conditions $f(\infty)=1, f(-\infty)=\lambda$ and seek a solution of the form $f(x, t)=F(x+c t)$. The equation for $F$ may be integrated once, giving

$c F+\frac{1}{F}+\gamma^{*} F^{\prime \prime \prime}=1+c$,

using the boundary condition at $+\infty$. The condition at $-\infty$ gives the relation $c=1 / \lambda$; thus $c>0$, and if waves exist they travel from right to left. Multiplying through by $F^{\prime}$ and integrating again we find

$\gamma^{*} \int_{-\infty}^{\infty}\left(F^{\prime \prime}\right)^{2} \mathrm{~d} x=\log c+\frac{1}{2 c}\left(1-c^{2}\right)$.

The left-hand side is clearly positive, and the righthand side is positive for $0<c<1$ and negative for $c>1$. Hence a necessary condition for a nontrivial travelling wave solution is $0<c<1$, i.e., $\lambda>1$, which we might have anticipated from Figs. 9-11. We can find numerical solutions to Eq. (48) together with the boundary conditions $F \rightarrow 1$ as $x \rightarrow \infty, F \rightarrow \lambda$ as $x \rightarrow-\infty$, using a shooting method. The result of such a calculation is shown in Fig. 12 (crosses), together with the long-time behaviour of a time-dependent simulation like that of Fig. 10. Here, larger values of $\gamma^{*}$ and $\lambda$ than 


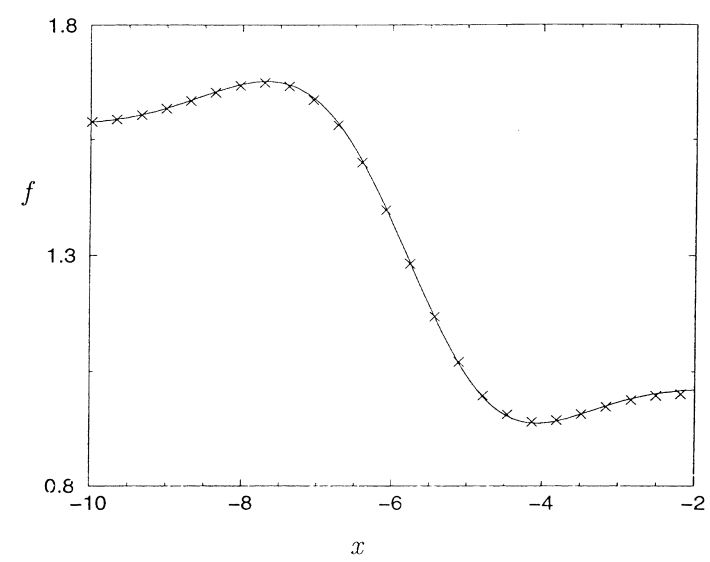

Fig. 12. Convergence to a travelling wave solution. The travelling wave solution has been plotted with crosses while the long-time behaviour of the solution to (45), with a ramp profile for initial data, is shown by the solid line. Here $\gamma^{*}=0.1$, $\lambda=1.6$.

those of Fig. 10 are used, for purposes of illustration, but clearly the main features of the evolution are identical. In particular, it is easily checked from Fig. 10 that the wavefront is moving with a speed close to the expected value of $0.666=1 / \lambda$.

\section{Conclusion}

Void electromigration is a Laplacian free boundary problem. To treat it we have adapted two analytical methods standard in Hele-Shaw flow: the conformal mapping and the 'shallowwaves' methods. With the first one, we focus on steady voids travelling in the middle of the strip or at the boundary. Since we have found that no steady solutions can travel along the boundary, we have examined the case of time-dependent solutions. We derive a simplified equation by asymptotic analysis. It turns out, that this equation develops finite-time singularity in absence of capillarity. With capillarity, well-behaved solutions are obtained.

\section{References}

[1] E. Arzt, O. Kraft, W.D. Nix, J.E. Sanchez Jr., J. Appl. Phys. 76 (1994) 1563.

[2] M. Ben Amar, Physica D 134 (1999) 275.

[3] O. Kraft, E. Arzt, Appl. Phys. Lett. 66 (1995) 2063.

[4] J.W. McLean, P.G. Saffman, J. Fluid Mech. 102 (1981) 455.

[5] M. Mahadevan, R.M. Bradley, J. Appl. Phys. 79 (1996) 6840.

[6] T. Maxworthy, J. Fluid Mech. 173 (1986) 95.

[7] J.H. Rose, Appl. Phys. Lett. 61 (1992) 2171.

[8] P.G. Saffman, G.I. Taylor, Proc. R. Soc. London Ser. A 245 (1958) 312.

[9] P.G. Saffman, J. Fluid Mech. 173 ( 1986) 73.

[10] G.M. Homsy, Annu. Rev. Fluid Mech. 19 (1987) 271.

[11] J.E. Sanchez Jr., O. Kraft, E. Arzt, Appl. Phys. Lett. 61 (1992) 3121

[12] M. Schimschak, J. Krug, Phys. Rev. Lett. 78 (1997) 278.

[13] M. Schimschak, J. Krug, Phys. Rev. Lett. 80 (1997) 1674.

[14] Z. Suo, W. Wang, M. Yang, Appl. Phys. Lett. 64 (1994) 1944.

[15] S. Tanveer, Phys. Fluids 29 (1986) 3537.

[16] S. Tanveer, Phys. Fluids 30 (1987) 651.

[17] R. Combescot, T. Dombre, Phys. Rev. A 38 (1988) 2573.

[18] W. Wang, Z. Suo, T.H. Hao, J. Appl. Phys 79 (1996) 2394. 